\title{
The Opacity of Spiral Galaxies from Counts of Distant Background Galaxies
}

\author{
B.W. Holwerda ${ }^{1,2}$, R.A. González ${ }^{3}$, R.J. Allen ${ }^{1} \&$ P.C. van der Kruit ${ }^{2}$ \\ (1) Space Telescope Science Institute, Baltimore, MD 21218, USA \\ (2) Kapteyn Institute, Landleven 12, 9747 AD Groningen, The \\ Netherlands \\ (3) Centro de Radioastronomía y Astrofísica, Universidad Nacional \\ Autónoma de México, 58190 Morelia, Michoacán, Mexico
}

\begin{abstract}
We have applied the "Synthetic Field Method" on a sample of 20 nearby galaxies in order to determine the opacity of their disks. We present preliminary results on the radial dependence of cold dust absorption for 3 examples. The spirals NGC 4535 and NGC 4725 show significant absorption at a half-light radius. UGC 2302, a LSB galaxy, shows much less opacity.
\end{abstract}

\section{Introduction}

The Synthetic Field Method (SFM) was developed by González et al. (1998) as a technique for determining the average opacity through the disk of a nearby spiral by counting more distant background galaxies. This number is compared with those of "synthetic fields", a known WFPC2 deep field, dimmed by a certain opacity, added to the foreground galaxy image.

González et al. (2003) examined the application of the method and concluded that the optimum results with HST/WFPC2 imaging would be obtained on Fornax and Virgo cluster galaxies, with the accuracy degrading for galaxies much more closer or distant.

Our sample was drawn from the HST archives, in part from the Cepheid Distance Scale HST Key project sample.

\section{Results}

We present preliminary results on NGC 4535, NGC 4725 and UGC 2302. The numbers of real and simulated field galaxies were compared in annuli of deprojected radius.

\subsection{NGC 4535 and NGC 4725}

NGC 4535 is a face-on $\mathrm{SABc}$ with a half-light radius of $5.1 \mathrm{kpc}$ at a distance of $16 \mathrm{Mpc}$. Several spiral arms with bright HII regions are visible in the HST image. NGC 4725 is a SABab ringed galaxy at $12 \mathrm{Mpc}$. Its half-light radius is 

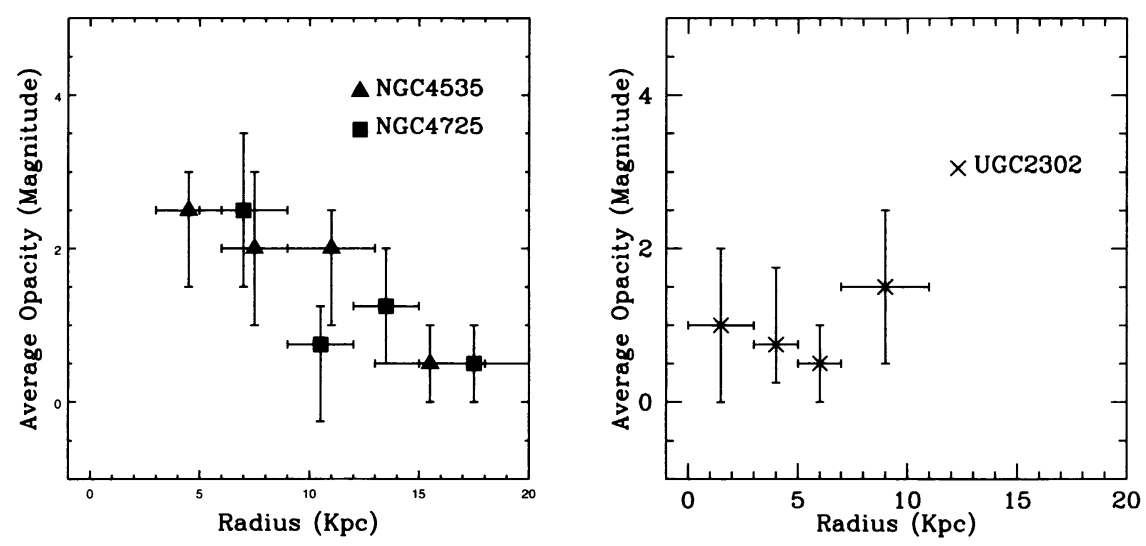

Figure 1. The radial opacity measurement from field galaxy counts for NGC 4535 and NGC 4725.

Figure 2. The radial opacity measurement of UGC 2302.

$5.4 \mathrm{kpc}$. The HST image shows much less structure. The radial opacity profiles of both however are similar (see Figure 1), showing 2.5 magnitudes of extinction in $I$ at the half-light radius, dropping off to 0.5 magnitude.

\subsection{UGC 2302}

UGC2302, a LSB at $15 \mathrm{Mpc}$, is a much more compact object. At comparable radii, it does not show as much absorption; less then a magnitude at $5 \mathrm{kpc}$ from the center.

\section{Conclusions}

The uncertainties in these individual profiles are large due to low numbers of field galaxies and an added uncertainty due to their clustering. By averaging a number of fields we will improve on these uncertainties for an average radial opacity model of spiral galaxies. For different Hubble types, we intend to compare the radial dependence of opacity with the HI density profile to obtain cold dust-to-gas ratios.

\section{References}

González, R. A., Allen, R. J., Dirsch, B., Ferguson, H. C., Calzetti, D., \& Panagia, N. 1998, ApJ, 506, 152

González, R. A., Loinard, L., Allen, R. J., \& Muller, S. 2003, AJ, 125, 1182

Holwerda, B. W., Allen, R. J., \& van der Kruit, P. C. 2002, ASP Conf. Ser. 273,337 